\title{
ANDES

www.scielo.cl

\section{Cuidados del desarrollo en recién nacidos prematuros: fundamentos y características principales}

\section{Developmental care of premature newborns: fundamentals and main characteristics}

\author{
Lisseth Barra C. ${ }^{\mathrm{a}, \mathrm{b}, \mathrm{c}}$, Alejandra Marín P. ${ }^{\mathrm{a}, \mathrm{c}}$, Soledad Coo ${ }^{\mathrm{b}}$ \\ aDepartamento Kinesiología, Facultad de Medicina. Universidad de Chile. Santiago, Chile

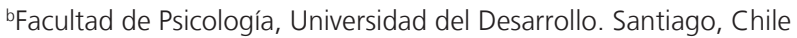 \\ cKinesióloga
}

Recibido: 16 de junio de 2020; Aceptado: 10 de septiembre de 2020

¿Qué se sabe del tema que trata este estudio?

Los recién nacidos prematuros extremos permanecen un largo período de tiempo en el Servicio de Neonatología donde, además de otorgar cuidados para la sobrevida del bebé, poco a poco se han ido incorporando cuidados centrados en el desarrollo.
¿Qué aporta este estudio a lo ya conocido?

El artículo realiza una síntesis actualizada de los fundamentos neurobiológicos que explican la importancia de prevenir/disminuir el estrés en recién nacidos prematuros, así como de la teoría y principales características de los cuidados centrados en el desarrollo.

\section{Resumen}

Los recién nacidos prematuros (RNP), en especial los extremos, requieren ser atendidos en las distintas unidades existentes en los servicios de neonatología. Además de procurar la sobrevida de estos niños, poco a poco estos servicios han ido incorporando intervenciones para promover su desarrollo. Esta revisión aporta una síntesis actualizada de los cuidados neonatales centrados en el desarrollo (CCD). En su primera parte describe los fundamentos teóricos de tipo neurobiológicos que explican cómo el denominado "estrés neonatal" puede afectar el curso de desarrollo de un niño nacido prematuro. Posteriormente, se revisa la Teoría Sinactiva, que es uno de los modelos teóricos que contribuye a entender las características de los CCD. Respecto de estos últimos, se describen y abordan sus evidencias y desafíos para la implementación desde una mirada crítica, destacando sus fortalezas y debilidades. Este artículo contribuye a destacar la importancia de seguir avanzando en la mejora de la calidad de la atención que reciben los RNP para promover la resiliencia y/o el mejor potencial de desarrollo posible en estos niños, además de relevar el rol de los padres en el cuidado neonatal.
Palabras clave:

Recién Nacido

Prematuro;

Neonatología;

Cuidados Centrados en

el Desarrollo;

Estrés Neonatal;

Teoría Sinactiva

Correspondencia:

Lisseth Barra C.

lisseth@uchile.c 


\begin{abstract}
Premature newborns, especially the extreme ones, need to be cared for in the different units in the neonatal services. In addition to ensuring the survival of these newborns, these services have gradually incorporated interventions to promote their development. This review provides an updated synthesis of developmental-focused neonatal care. First, it describes the neurobiological theoretical foundations that explain how the so-called "neonatal stress" can affect the development of a premature child. Later, it reviews the Synactive Theory, which is one of the theoretical models that contribute to understanding the characteristics of the care focused on development. Regarding this care, the evidence and challenges for implementing are described and addressed from a critical perspective, pointing out the strengths and weaknesses. This article highlights the importance of improving the quality of care that preterm newborns received in NICUs, promoting resilience and/or the best possible developmental potential in these children, in addition to strengthening the role of parents in neonatal care.
\end{abstract}

\section{Keywords:}

Premature Newborn; Neonatology Services; Development-Centered Care; Neonatal Stress; Sinactive Theory

\section{Introducción}

Cada año nacen 15 millones de prematuros en el mundo ${ }^{1}$. La prematuridad es la principal causa de morbilidad perinatal en los países desarrollados ${ }^{1}$, lo que demanda a los equipos de salud neonatales a preocuparse de la sobrevida de estos bebés, sin olvidar su calidad de vida a largo plazo. Esto requiere una gran experticia de los cuidados ofrecidos en las unidades de cuidados intensivos neonatales (UCIN).

De acuerdo con la Organización Mundial de la Salud son prematuros aquellos recién nacidos $(\mathrm{RN})$ que nacen antes de 37 semanas de gestación ${ }^{2}$. En Chile un $8,2 \%$ de los $\mathrm{RN}$ son prematuros, y de estos, un $1,2 \%$ corresponde a aquellos considerados extremos ${ }^{3}$. La prematuridad, en especial la extrema, implica que los niños nacen antes de completar las etapas necesarias de maduración intrauterina y requieren ser atendidos en Servicios de Neonatología, en las unidades de cuidado intensivo neonatal (UCIN). Inicialmente, el único objetivo de estas unidades era ofrecer atención profesional para lograr la sobrevida del recién nacido prematuro (RNP). En las últimas décadas, el cuidado profesional otorgado en la UCIN también ha buscado mejorar la calidad de la atención y promover un mejor desarrollo para sus pacientes. Con este objetivo se ha incorporado el modelo de Cuidados Centrados en el Desarrollo (CCD), lo que ha implicado la incorporación de un nuevo estándar de atención dirigido a los niños de alto riesgo, quienes son en su mayoría prematuros ${ }^{4}$.

La sobrevida de los prematuros de muy bajo peso en nuestro país ha mejorado significativamente en las últimas décadas, debido a los avances en la medicina perinatal y el progreso de servicios de neonatología. Estos son servicios altamente organizados que otorgan cuidados en diferentes niveles de complejidad a neonatos con patología han ido incluyendo, en la práctica clínica, estrategias de cuidados del neurodesarrollo ${ }^{5,6}$. Aunque, estas han sido poco documentadas, ha sido posible encontrar algunas experiencias de lo que ocurre a nivel nacional ${ }^{7-9}$.

Para facilitar la definición de estándares en los servicios de neonatología que contribuyan a los CCD, es necesario que los profesionales del área comprendan claramente el concepto, así como sus fundamentos y sus implicancias para el modelo de atención. ¿Cuáles son los fundamentos teóricos que justifican la importancia de los cuidados perinatales enfocados al desarrollo del RNP? ¿Cuáles son las características principales de los CCD? En este artículo se describirán los fundamentos teóricos que justifican la pertinencia de dichos cuidados, así como las características principales de los CCD. El objetivo principal de esta revisión es analizar de manera reflexiva y crítica la relevancia de los cuidados de desarrollo en etapa perinatal en el RNP, como estrategia fundamental para lograr el mayor potencial de desarrollo en estos niños.

\section{Fundamentos teóricos neurobiológicos}

La sobrevida de los RNP ha aumentado, pero las secuelas motoras, sensoriales o cognitivas como consecuencia de la prematuridad siguen siendo un problema importante ${ }^{10,11}$. Las causas del deterioro del neurodesarrollo en los RNP son multifactoriales e incluyen hipoxemia, enfermedad crítica y lesiones cerebrales perinatales. Además de estos factores importantes, la exposición al estrés en el contexto de hospitalización es un determinante crítico de los resultados del desarrollo neurológico más allá de lo predicho por otros factores ${ }^{12}$.

Los RNP ingresados en la UCIN están expuestos a importantes factores ambientales estresantes, incluidos: manipulaciones asociadas a procedimientos médicos, procedimientos dolorosos, altos niveles de ruido 
y luz, entre otros. Los investigadores han relacionado la exposición al estrés en la UCIN con alteraciones a largo plazo en la respuesta al estrés y deterioro del desarrollo neurológico ${ }^{12-14}$. Los niños prematuros que ingresan en las UCIN poseen un cerebro inmaduro, donde aún están migrando neuronas a la corteza y formando miles de sinapsis nuevas ${ }^{15}$. Por consiguiente, la organización de nuevas conexiones dependerá en parte de los estímulos recibidos durante su estadía hospitalaria.

La neurobiología explica diversos mecanismos mediante los cuales el estrés puede afectar el neurodesarrollo. Estos pueden sintetizarse utilizando el modelo de incrustación de estrés neonatal (Neonatal Stress Embedding Model), que describe los mecanismos neurobiológicos de la incrustación biológica por exposición al estrés neonatal en la UCIN y sus efectos en el desarrollo neurológico. La programación biológica es el proceso por el cual la exposición temprana al estrés, durante períodos críticos o sensibles del desarrollo, afecta los resultados de salud a largo plazo a través de cambios permanentes en los procesos biológicos ${ }^{16}$. Los períodos críticos o sensibles son aquellos durante los cuales se produce un crecimiento rápido del cerebro, lo que lo hace vulnerable a daños ambientales. Los cambios en los procesos biológicos pueden ocurrir mediante la reprogramación de los parámetros funcionales de los sistemas biológicos o mediante marcas epigenéticas heredables ${ }^{15}$. De acuerdo al modelo de incrustación neonatal, en los RNP existen 4 tipos de mecanismos neurobiológicos involucrados que pueden explicar la inclusión biológica de la exposición al estrés durante la hospitalización en la UCIN: la reprogramación del sistema inmune, del sistema nervioso autónomo y del eje hipotalámico-pituitario-adrenal (HPA), así como los cambios en la expresión génica ${ }^{12}$. Respecto de este último tipo de mecanismo, hay un modelo teórico para la investigación de epigenética conductual en prematuros, recientemente desarrollado ${ }^{17}$.

Por otra parte, el modelo de incrustación neonatal del estrés también considera el impacto de la experiencia prenatal en los resultados del desarrollo, es decir, de los mecanismos de programación fetal; respecto de los cuales los estudios han demostrado que el estrés severo fetal en el primer semestre del embarazo se asocia con menor peso y edad gestacional al nacer y también a menor desarrollo ${ }^{18}$. Esto nos sugiere que, en el caso de algunos prematuros, el efecto del estrés pudiera ser acumulativo debido a la carga de estrés prenatal y el distrés experimentado en la UCIN.

Los mecanismos neurobiológicos señalados fundamentan la necesidad de disminuir el estrés que experimentan los RNP en las UCIN, que es uno de los principales objetivos de los CCD. Sin embargo, desde una perspectiva multinivel, también pueden encontrarse fundamentos teóricos relevantes desde el propio de- sarrollo infantil. En relación a este punto, es relevante considerar la Teoría Sinactiva, que se describe a continuación.

\section{Teoría sinactiva}

En 1982, Heidelise Als propuso la Teoría Sinactiva o "interactiva" (synactive theory) que es un modelo teórico del desarrollo integrador, orientado a sistemas y basado en relaciones. Esta teoría propone que el desarrollo del cerebro infantil procede en una secuencia ordenada con diferenciación de comportamientos evidentes con la maduración. Identifica subsistemas superpuestos que proporcionan la base para comprender el comportamiento observado en bebés vulnerables ${ }^{19}$. La teoría postula que los recién nacidos humanos forman activamente su propio desarrollo, y que éste está mediado por su capacidad de atención-interacción y su relación con el entorno y cuidadores. Para poder interpretar la conducta se debe valorar cuatro subsistemas jerarquizados ${ }^{20}$. El primero es el subsistema nervioso autónomo, que regula el funcionamiento fisiológico básico necesario para sobrevivir; este dirige e impacta a los otros subsistemas, siendo más preponderante a menor edad gestacional. El segundo subsistema, el motor, se manifiesta en el tono, la actividad y la postura. El tercer subsistema se refiere a los estados de vigilia y sueño. El cuarto es atención-interacción, que es la capacidad del recién nacido de interactuar con el medio y mantener la alerta. Finalmente, se considera la autorregulación, que tiene como función mantener el balance entre los cuatro subsistemas mencionados y que se presenta a partir de las 32-35 semanas de edad gestacional $^{21,22}$. De acuerdo a esta teoría, cuando un $\mathrm{RN}$ presenta comportamientos que reflejan signos de estrés, se considera que se encuentra desorganizado. En cambio, si presenta signos de autorregulación, se considera organizado.

Esta teoría ha proporcionado el principal soporte teórico para la comprensión de señales infantiles a través del estado fisiológico y comportamientos motores y la consecuente práctica de cuidados del desarrollo ${ }^{23}$. En otras palabras, esta teoría constituye la base sobre la cual se ha desarrollado el modelo de CCD que, en esencia, es un enfoque de cuidados basados en una evaluación continua del bebé, monitoreando frecuentemente las capacidades de los subsistemas mencionados. En el siguiente apartado se describe la aplicación práctica de este modelo.

\section{Cuidados centrados en el desarrollo y la familia}

Los CCD aplicados a los RN ingresados en neonatología son una serie de intervenciones profesionales basadas en la interpretación de la conducta del bebé y 
en la implementación de estrategias para disminuir su estrés, favoreciendo su desarrollo neurológico y emocional y facilitando la integración de la familia como cuidadores. El modelo implica que las intervenciones dirigidas a los RNP deben ser adecuadas a su edad gestacional (asociada al grado de su maduración cerebral) y condición de salud, proporcionando un ambiente similar al intrauterino. Cuando las intervenciones en el RNP se organizan colaborativamente en la UCIN con un protocolo de atención específico a sus necesidades, podemos hablar de cuidados centrados en el desarrollo individualizado, denominado Neonatal Individualised Developmental Care and Assessment Programme (NIDCAP) que es el modelo específico propuesto por Als y, también, el programa de CCD más ampliamente difundido.

La conducta es el principal canal de comunicación de los RNP durante su estadía en la UCIN. Por ello, en los CCD es clave valorar y comprender su significado. El RN guía al cuidador y es considerado un participante activo en los cuidados que recibe. Esta orientación, en la práctica clínica, requiere tanto sensibilidad como presencia y vigilancia por parte del equipo de salud, para reconocer y apreciar los diferentes matices de respuesta que permitan realizar las intervenciones necesarias. De este modo, todas las interacciones que involucran al bebé (actividades de cuidado diario, procedimientos médicos o interacción simple) se basan en responder a sus necesidades, respetando su descanso o recuperación después de un evento estresante (ej. extracción de sangre).

El modelo de CCD posee las siguientes líneas de acción: Optimizar tanto el macroambiente como el microambiente en el que se desarrolla el niño e involucrar a la familia para potenciar su papel de cuidador principal de manera activa y continua. Las intervenciones del macroambiente implican estrategias para reducir la presencia ruidos y luces, así como modulan otros elementos de la sala en la UCIN. El cuidado del microambiente promueve una postura de flexión fisiológica, cambios de posición pertinentes, así como modular las manipulaciones y maniobras específicas para disminuir el dolor, entre otras. En cuanto a la familia, se utilizan distintas estrategias que cambian la estructura de las Unidades. Esto se expresa en la flexibilización de horarios de visita y, sobre todo, en las actitudes del equipo, que toman conciencia de que tratan a familias y no solo a RN, por lo que se precisa apertura a los padres las $24 \mathrm{~h}$ del día para facilitar la lactancia materna y el cuidado canguro. Hay numerosos estudios que describen y profundizan sobre las estrategias que utilizan estas líneas de acción señaladas y revisiones que las sintetizan $^{22}$. Sin embargo, un aspecto menos descrito es el acompañamiento emocional que se otorga a los padres.
Estudios señalan que las necesidades emocionales de los padres, en particular de las madres, son invisibilizadas ante las demandas relacionadas a la salud y el esfuerzo de los profesionales por lograr la sobrevida del niño ${ }^{24}$. Los CCD promueven las interacciones padres-hijos, pues es importante desarrollar lazos emocionales saludables para el proceso de apego. La participación de los padres en las UCIN contribuye a que estos muestren menores niveles de ansiedad y desarrollen confianza en el cuidado de sus hijos, así como una mayor atención a sus necesidades. La forma en que se realiza el acompañamiento de los padres no se describe detalladamente en los estudios centrados en los CCD, sin embargo, el enfoque de salud mental infantil iniciado por Selma Fraiberg nos ejemplifica de manera práctica cómo los profesionales que trabajan en la UCIN pueden apoyar la salud mental de padres e hijos. Este abordaje es preventivo y terapéutico, culturalmente sensible y orientado a las relaciones, basado en la psiquiatría y psicoanálisis ${ }^{25}$. Este enfoque es más amplio que la propuesta de CCD realizada en la UCIN, puesto que el modelo de acompañamiento emocional y educativo de Fraiberg abarca desde el embarazo hasta los de tres años, atendiendo a familias con niños de alto riesgo.

En cuanto a la evaluación de la eficacia de la aplicación de CCD, esta presenta resultados ambivalentes. Los estudios han encontrado que los RNP tratados con este modelo tienen menos necesidad de procedimientos médicos, mejores resultados de comportamiento en etapas claves, inicio temprano de la alimentación oral, mejor incremento de peso diario promedio y estancias hospitalarias más cortas, entre otros resultados favorables $^{22}$. Por otro lado, la eficacia del programa NIDCAP para mejorar variables médicas a corto plazo y del neurodesarrollo del prematuro a largo plazo no ha sido demostrada en estudios de meta-análisis ${ }^{26}$. Sin embargo, varios autores, entre ellos la autora del modelo y otros que trabajan con CCD (concepto más amplio que el modelo NIDCAP), han defendido el modelo NIDCAP, basándose en la dificultad para estudiar una gran gama de abordajes y cambios del comportamiento, cuyo nivel de intervención no está estandarizada. Por esta razón, los diseños tipo ensayos aleatorizados convencionales son difíciles de desarrollar. Además, apuntan a otras deficiencias metodológicas existentes en el estudio de Ohlsson \& Jacobs, entre ellas la confusión de estrategias dado que incluyen instrumentos y estrategias que no son del modelo, y la combinación de estudios que no son comparables debido a que la población de prematuros es heterogénea ${ }^{26-28}$. Aun considerando estas observaciones, Ohlsson \& Jacobs mantienen sus conclusiones iniciales, respondiendo que no hay evidencia sustantiva para recomendar la implementación del NIDCAP en su forma actual como el modelo de 
atención estándar en bebés prematuros ${ }^{26,29}$. Pese a esto, y al posible conflicto de interés por parte de quienes promueven el método NIDCAP, la evidencia clínica producto de metodologías cualitativas indica que los cuidados NIDCAP y otras prácticas propias del CCD cumplirían una función fundamental en la humanización de los cuidados que se realizan en las $\mathrm{UCIN}^{30,31}$.

\section{Desafíos en la implementación de los CCD}

La implementación de los CCD implica hacer cambios importantes en las rutinas de trabajo de las UCIN y en la actitud de los equipos de salud hacia sus pacientes. El aspecto central del CCD es que los procesos se deben ajustar a las capacidades y necesidades de los RNP. Por consiguiente, los procedimientos se adaptan a las características del niño y su familia, es decir, las intervenciones dirigidas a los bebés deben ajustarse a las capacidades y necesidades de ellos, y no a la comodidad $\mathrm{u}$ horarios que prefieran los profesionales en neonatología.

Este tipo de cuidados se realiza en muchos países europeos desde hace algunas décadas. Sin embargo, su aplicación en Latinoamérica ha sido menos documentada. Un estudio realizado con el objetivo de describir el grado de implementación de los CCD en las UCIN de México concluyó que faltaban estrategias para elevar la cobertura de cuidados centrados en el desarrollo en las UCIN, además de mejorar la educación de los padres respecto a las medidas utilizadas en él y lograr que los cuidados centrados en el desarrollo sean aplicados en todas las regiones de dicho país ${ }^{32}$.

Cabe señalar que algunas de las estrategias de los CCD son de bajo costo y fácil implementación, a diferencia del NIDCAP que es un programa que debe contar con profesionales formados en el modelo, lo que dificulta su implementación ${ }^{22}$. En los servicios de neonatología en Chile se considera un espacio para los padres en la planta física y, dentro de los recursos humanos, a kinesiólogos para atención respiratoria y de neurorrehabilitación ${ }^{6}$. Aunque no se mencionan explícitamente estándares en la línea de los CCD, hay antecedentes que hacen notar que la preocupación por el desarrollo, como se señaló anteriormente, se ha ido incorporando a la práctica clínica de la neonatología chilena.

Desde el año 2001, la Sociedad Chilena de Pediatría y miembros asociados, firmaron la declaración de $\mathrm{Pu}$ cón ${ }^{33}$, la cual adhiere a la "iniciativa de humanización de cuidados neonatales (IHCN)" 34 . La humanización de la pediatría perinatal tiene varios elementos que se corresponden con los CCD, entre ellos la atención dirigida a los padres y la promoción de su participación activa en los cuidados del niño en la UCIN, además de la disminución del estrés del bebé mediante disminuir la cantidad de exámenes y de análisis de laboratorio y las terapias agresivas. En línea con lo anterior, se han documentado experiencias de trabajo en CCD bajo la denominación de cuidados humanizados ${ }^{7-9}$. Algunos hospitales han desarrollado guías de práctica clínica que incorporan la temática de los cuidados del desarrollo ${ }^{35-37}$. Por tanto, aunque está poco documentado, en la práctica, los servicios de neonatología de Chile han ido incorporando estrategias de CCD, tales como el método canguro, cuidados posicionales y protocolos de mínima manipulación. En estos cuidados individualizados del desarrollo, participan distintos profesionales, incluyendo enfermeras, kinesiólogos y terapeutas ocupacionales ${ }^{35-37}$.

La reflexión sobre los cambios organizacionales, actitudinales y las estrategias de los CCD descritas en la literatura sugieren que éstas pueden aplicarse en las UCIN, incluso en las unidades sin la formación en el Programa NIDCAP. El alto costo de la formación en este programa no debiese ser un impedimento para que las UCIN sigan avanzando hacia una mejor calidad y humanización de atención de los RNP en los servicios de neonatología. Esto debiera traducirse en que los estándares de calidad de dichos servicios incorporen cuidados que disminuyan el estrés neonatal y promuevan el desarrollo de los recién nacidos prematuros.

\section{Conclusiones y comentarios finales}

Considerando lo revisado, podemos concluir que existen fundamentos neurobiológicos que explican claramente los efectos perjudiciales del estrés sobre la estructura y función del sistema nervioso del RNP. Además, cuenta con un modelo teórico del desarrollo, la Teoría Sinactiva de $\mathrm{Als}^{20,21}$, que es un modelo sistémico, interactivo, y por tanto coherente con los conocimientos del desarrollo. Por consiguiente, dado que los CCD pretenden disminuir el estrés y el sufrimiento del niño, así como también favorecer su desarrollo promoviendo la integración de los padres como cuidadores, se puede concluir que este enfoque cuenta con respaldo teórico sustentable para su aplicación. Existen suficientes argumentos que justifican la utilización de los CCD en los servicios de neonatología: estudios que respaldan las estrategias utilizadas, entre ellos los resultados del seguimiento de RNP, argumentos éticos relacionados al bienestar y humanización de la atención clínica y la satisfacción de los padres y profesionales, además de los aspectos económicos.

Pese a que se cuenta con elementos suficientes para promover la utilización de los CCD, se requieren estudios que contribuyan a esclarecer las evidencias científicas del modelo; mejorando las estandarizaciones de las intervenciones, utilizando distintos tipos y diseños 
de investigación. Sería relevante incluir más investigación cualitativa para valorar la experiencia subjetiva de los padres en relación con las demandas de tener un hijo en neonatología y de cómo el modelo de CCD los beneficia, o para conocer necesidades emocionales de ellos que aún no han sido cubiertas por este modelo. Además, la participación de los padres y madres en la UCIN y la recuperación del rol como cuidadores que promueven los CCD, no debe justificarse sólo desde la evidencia científica, sino también desde una perspectiva de derecho.

\section{Conflicto de intereses}

Los autores declaran no tener conflicto de intereses.

\section{Referencias}

1. Arroyo V, Linares C, Díaz J. Premature births in Spain: Measure the impact of air pollution using time series analyses. Scien Environment. 2019;660:105-14.

2. Althabe F, Howson CP, Kinney M, Lawn J \& World Health Organization. Born too soon: The global action report on preterm birth. 2012; Recuperado de http://www.who.int/pmnch/media/ news/2012/201204\%5Fborntoosoonreport.pdf.

3. Ministerio de Salud de Chile. Departamento de Estadística e Información en Salud. (2016). Nacimientos inscritos según edad gestacional. 2016. Recuperado el 25 de junio de 2019, de Tableau Software website: https://public.tableau.com/views/ Anuario_Nacimientos_3_final/Nacim ientos?:embed $=y \&$ :showVizHome $=$ no \&:host_url=https\%3A\%2F\%2Fpublic. tableau.com\%2F\&:embed_code_ve rsion $=3 \&:$ tabs $=$ no\&:toolbar $=$ yes $\&$ :animate_transition=yes\&:display_ static_image $=$ no\&:display spinner $=$ no\&:display_ overlay=yes\&:display_count=yes\&publish $=$ yes $\&$ :loadOrderID $=0$.

4. Gibbins S, Hoath SB, Coughlin M, Gibbins A, Franck L. The Universe of Developmental Care: A New Conceptual Model for Application in the Neonatal Intensive Care Unit. Adv Neo Care. 2008;8(3):141.

5. Hübner GME, Ramírez FR. Sobrevida, viabilidad y pronóstico del prematuro. Rev Med Chile 2002;130:931-8.

6. Novoa JM, Milad M, Vivanco G, Fabres J, Ramírez R. Recomendaciones de organización, características y funcionamiento en Servicios o Unidades de Neonatología. Rev Chil Pediat. 2009;80(2).

7. Martínez JL, Villalón H. Cuidados humanos en neonatología, y una nueva forma de enfocar al recién nacido y su familia. Rev Med Clin Condes. 2008;19(3):159-64.

8. Martínez JL, Villalón H. Cuidados Humanos en Neonatología. Modelo en un Centro Privado. XLII Congreso Chileno de Pediatría. Rev. Chil. Pediatr. 2002; vol. $73 n^{\circ} 6$.

9. Villalón H, Martínez JL, Agurto A, Villalobos J, Aguila P. Estimulación temprana sensorio - motriz centrada en la familia, en el prematuro extremo. XLV Congreso Chileno de Pediatría. Rev Chil Pediatr 2005;76:83.

10. Brydges R, Landes K, Reid L, Campbell C, French N, Anderson M. Cognitive outcomes in children and adolescents born very preterm: A meta-analysis. Develop Med Child Neurol. 2018;60:45268.

11. Howe TH, Sheu CF, Hsu YW, Wang TN, Wang LW. Predicting neurodevelopmental outcomes at preschool age for children with very low birth weight. Res Develop Disab. 2016;48:231-41.

12. Nist MD, Harrison TM, Steward DK. The biological embedding of neonatal stress exposure: A conceptual model describing the mechanisms of stressinduced neurodevelopmental impairment in preterm infants. Res Nurs Health. 2019;42(1):61-71.

13. Brummelte S, Chau C, Cepeda I, Degenhardt A, Weinberg J, Synnes AR, et al. Cortisol levels in former preterm children at school age are predicted by neonatal procedural pain-related stress. Psychoneuroendocrinology. 2015;51:15163.

14. Grunau RE, Cepeda IL, Chau CMY, Brummelte S, Weinberg J, Lavoie PM, et al. Neonatal Pain-Related Stress and NFKBIA Genotype Are Associated with Altered Cortisol Levels in Preterm Boys at School Age. PLoS ONE, 2013;8(9).

15. Buschdorf P, Meaney J. Epigenetics/ Programming in the HPA Axis. Compr Physiol. 2015;6:87-110.

16. Nist MD. Biological embedding: Evaluation and analysis of an emerging concept for nursing scholarship. J Adv Nurs. 2017:73:349-60.

17. Provenzi L, Guida E, Montirosso R. Preterm behavioral epigenetics: A systematic review. Neuroscien Biobeh Rev. 2018;84:262-71.

18. Glover V. Maternal depression, anxiety and stress during pregnancy and child outcome; what needs to be done. Best Pract Res Clin Obstet gynecol. 2014;28:2535.

19. Shulman C. Theoretical Bases of Intervention in Infant and Early Childhood Mental Health. En C. Shuman (ed) Reseach and Practice in Infant and Early Chilhood Mental Health pp. 145-58.

20. Als H. Toward a synactive theory of development: Promise for the assessment and support of infant individuality. Inf Ment Health J. 1982;3:229-43.

21. Als H. A Synactive Model of Neonatal Behavioral Organization: Phys Occupat Ther Pediat. 1986;6:3-53.

22. Acevedo H, Becerra R, López Martínez A La filosofía de los cuidados centrados en el desarrollo del recién nacido prematuro (NIDCAP): Una revisión de la literatura. Enfermería Global. 2017;16:577-89.

23. Gibbins S, Hoath S, Coughlin M, Gibbins A, Franck L. The Universe of Developmental Care: A New Conceptual Model for Application in the Neonatal Intensive Care Unit. Adv Neo Care. 2008;8:141.

24. Fowler C, Green J, Elliott D, Petty J, Whiting $\mathrm{L}$. The forgotten mothers of extremely preterm babies: A qualitative study. J Clin Nurs 2019;28:2124-34.

25. Weatherston J, Browne JV. What Is Infant Mental Health and Why Is It Important for High-risk Infants and Their Families? NewInft Nurs Rev. 2016;16:259-63.

26. Ohlsson A, Jacobs E. (2013a). NIDCAP: A systematic review and meta-analyses of randomized controlled trials. Pediatrics. 2013;131:e881-93.

27. Als H. Re: Ohlsson and Jacobs, NIDCAP: A Systematic Review and Meta-analyses. Pediatrics. 2013:132:e552-e3.

28. Haumont D, Amiel-Tison C, Casper C, Conneman N, Ferrari F, Huppi P, et al. NIDCAP and Developmental Care: A European Perspective. Pediatrics 2013;132: e551-2.

29. Ohlsson A, Jacobs E. Authors' Response: NIDCAP: A Systematic Review and Metaanalyses of Randomized Controlled Trials. Pediatrics. 2013;132:e553-7.

30. Nelson AM, Bedford PJ. Mothering a Preterm Infant Receiving NIDCAP Care 
in a Level III Newborn Intensive Care Unit. J Pediat Nurs 2016;31:e271-82.

31. Gutiérrez-Padilla JA, Pérez-Rulfo ID, Angulo-Castellanos E, Valle-Delgado E, García-Hernández HA, MartínezVerónica R. Cuidados centrados en el desarrollo en unidades de neonatología de México, 2015. Encuesta a través de redes sociales. Ginecol Obstet Mex. 2017;6:35563.

32. Solhaug M, Torunn Bjørk I, Pettersen Sandtrø H. Staff Perception One Year After Implementation of the The
Newborn Individualized Developmental Care and Assessment Program

(NIDCAP). J Pediat Nurs. 2010;25:89-97.

33. Pinto L. Fernando. Declaración de Pucón XLI Congreso Chileno de Pediatría Pucón, Noviembre 28 de 2001. Andes pediatr. 2002;73:197.

34. Vieira M. La Iniciativa de Humanización de Cuidados Neonatales. Rev Chil Pediat 2003;74:197-205.

35. Castellanos M, Vascopé X, Bastías R. Asistencia individualizada del recién nacido. En Salvo H. Guías Clínicas de
Neonatología, Hospital Santiago Oriente, Hospital Luis Tisné. Cuarta Edición, Año 2020.

36. Muñoz C. Cuidados de enfermería centrados en el neurodesarrollo. En: Ararat F, Arriagada JP, Bravo T. y Cols. Guías de Práctica Clínica Unidad de Paciente Crítico Neonatal Hospital San Juan de Dios de La Serena. Quinta Edición, Año 2020.

37. Mira A, Bastías R. Terapia ocupacional neonatal, una propuesta para la acción. Rev Chil Ter Ocupac. 2006;2206:23-32. 